\title{
Article \\ Systematic Scoring of Tubular Injury Patterns Reveals Interplay between Distinct Tubular and Glomerular Lesions in ANCA-Associated Glomerulonephritis
}

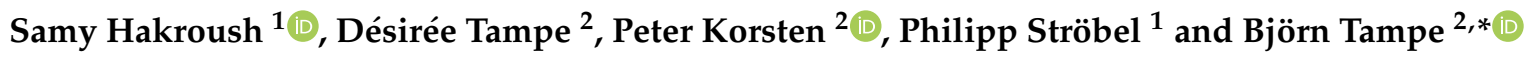 \\ 1 Institute of Pathology, University Medical Center Göttingen, 37075 Göttingen, Germany; \\ samy.hakroush@med.uni-goettingen.de (S.H.); philipp.stroebel@med.uni-goettingen.de (P.S.) \\ 2 Department of Nephrology and Rheumatology, University Medical Center Göttingen, \\ 37075 Göttingen, Germany; desiree.tampe@med.uni-goettingen.de (D.T.); \\ peter.korsten@med.uni-goettingen.de (P.K.) \\ * Correspondence: bjoern.tampe@med.uni-goettingen.de
}

check for updates

Citation: Hakroush, S.; Tampe, D.; Korsten, P.; Ströbel, P.; Tampe, B. Systematic Scoring of Tubular Injury Patterns Reveals Interplay between Distinct Tubular and Glomerular Lesions in ANCA-Associated Glomerulonephritis. J. Clin. Med. 2021, 10, 2682. https://doi.org/ $10.3390 / \mathrm{jcm} 10122682$

Academic Editor: Clive N. May

Received: 25 May 2021

Accepted: 15 June 2021

Published: 18 June 2021

Publisher's Note: MDPI stays neutral with regard to jurisdictional claims in published maps and institutional affiliations.

Copyright: (c) 2021 by the authors. Licensee MDPI, Basel, Switzerland. This article is an open access article distributed under the terms and conditions of the Creative Commons Attribution (CC BY) license (https:// creativecommons.org/licenses/by/ $4.0 /)$.

\begin{abstract}
Background: Antineutrophil cytoplasmic antibody (ANCA)-associated vasculitis (AAV) is a small vessel vasculitis, most frequently presenting as microscopic polyangiitis (MPA) or granulomatosis with polyangiitis (GPA). Acute tubular injury with the presence of tubulitis was previously reported to be of prognostic value in ANCA glomerulonephritis (GN). In particular, distinct tubular injury lesions were associated with the deterioration of kidney function at AAV disease onset, as well as renal resistance to treatment, and higher risk of progression to composite outcome in patients with AAV. To expand our knowledge regarding distinct tubular lesions in AAV, we aimed to describe acute tubular injury patterns in association with glomerular lesions in ANCA GN by systematic histological scoring. Methods: A total number of 48 renal biopsies with confirmed renal involvement of AAV admitted to the University Medical Center Göttingen from 2015 to 2020 were retrospectively examined. By systematic scoring of tubular injury lesions, the association between clinical parameters, laboratory markers, and histopathological findings was explored. Results: We have shown that cellular casts in renal biopsies were frequently observed in the majority of cases with ANCA GN. Furthermore, we showed that tubular epithelial simplification with dilatation correlated with MPA and MPO subtypes, C3c hypocomplementemia, severe renal involvement, and uACR. Red blood cell (RBC) casts were associated with increased levels of C-reactive protein (CRP), leukocyturia, and hematuria. Finally, we found that hyaline casts were associated with an increased fraction of glomeruli with global glomerular sclerosis. Conclusions: Acute tubular injury patterns were correlated with active ANCA GN, whereas tubular injury lesions reflecting the later stages of kidney disease correlated with chronic glomerular lesions. These results suggest an interplay between different renal compartments.
\end{abstract}

Keywords: systemic vasculitis; autoimmune diseases; anti-neutrophil cytoplasmic antibody; ANCA glomerulonephritis; acute tubular injury

\section{Introduction}

Antineutrophil cytoplasmic antibody (ANCA)-associated vasculitis (AAV) is a small vessel vasculitis that, according to the 2012 revised Chapel Hill Consensus Conference Nomenclature of Vasculitides, most frequently presents as microscopic polyangiitis (MPA) or granulomatosis with polyangiitis (GPA) [1,2]. Renal involvement is a common and severe complication of $\mathrm{AAV}$, potentially resulting in a pauci-immune necrotizing and crescentic ANCA glomerulonephritis (GN) with acute kidney injury (AKI), end-stage renal disease (ESRD), or death [2]. Clinicopathologic studies from the European Vasculitis Study Group (EUVAS) have demonstrated that distinct glomerular lesions are related to renal outcome in ANCA GN [3-6]. Derived from these studies, a histopathological subgrouping 
into four classes (focal, crescentic, mixed, and sclerotic), as defined by Berden et al., was shown to predict long-term renal survival rates [7]. These results were confirmed in multiple independent studies in recent years [8-24]. However, multivariable analyses could not demonstrate any improvement of outcome prediction in most of these studies, mainly attributed to the lack of outcome difference in the crescentic and mixed classes [16-27]. Therefore, Brix et al. suggested the ANCA renal risk score (ARRS) through the incorporation of tubular atrophy/interstitial fibrosis (TA/IF) to the percentage of normal glomeruli and baseline glomerular filtration rate (GFR) to predict ESRD in patients with AAV, underscoring the relevance of tubulointerstitial and glomerular lesions in ANCA GN [28]. Furthermore, acute tubular injury with the presence of tubulitis was previously reported to be of prognostic value in ANCA GN [4,5,29]. In particular, distinct tubular injury lesions were associated with the deterioration of kidney function at AAV disease onset, renal resistance to treatment, and a higher risk of progression to composite outcome in patients with AAV [30]. As a result of persistent tubular injury, TA/IF develops and further impairs kidney function, being characterized by a vicious circle of tubular epithelial damage, TA/IF, and chronic glomerular injury [31,32]. To expand our knowledge of distinct histomorphological tubular changes in AAV, we aimed to describe tubular injury lesions in association with glomerular lesions in ANCA GN by systematic histological scoring [33].

\section{Materials and Methods}

\subsection{Study Population}

A total number of 48 renal biopsies with confirmed renal involvement of AAV admitted to the University Medical Center Göttingen from 2015 to 2020 were retrospectively examined. The patient cohort has, in part, been previously described [34-37]. Medical records were used to obtain data on the age, sex, diagnosis (MPA or GPA), and laboratory results. The estimated glomerular filtration rate (GFR) was calculated using the Chronic Kidney Disease Epidemiology Collaboration (CKD-EPI) equation [38]. The worst measurement during the initial course of the disease was used to determine the severity of kidney injury.

\subsection{Definitions}

At admission, the Birmingham Vasculitis Activity Score (BVAS) version 3 was calculated as described previously [39]. The BVAS is assessed on a scale from 0 to 63, with a score of 0 indicating the absence of disease activity and higher scores indicating active disease. The simplified acute physiology score (SAPS) II was calculated according to the published guidelines [40]. RRT was performed intermittently in all cases, indications of RRT included serum creatinine $\geq 5.8 \mathrm{mg} / \mathrm{dL}(\geq 500 \mu \mathrm{mol} / \mathrm{L})$, severe electrolyte or acid-base abnormalities, and volume overload or uremic encephalopathy. RRT was terminated when the GFR surpassed $15 \mathrm{~mL} / \mathrm{min} / 1.73 \mathrm{~m}^{2}$ in the absence of hyperkalemia, heart failure, edema, or uremic encephalopathy.

\subsection{Urinary Analysis}

Levels of total proteinuria, urinary albumin, immunoglobulin $G(\operatorname{IgG}), \alpha_{1}$-microglobulin, and $\alpha_{2}$-macroglobulin were normalized to urinary creatinine concentration to control for variations in urine flow rate [41]. Leukocyturia and hematuria per high-power field (HPF) were semiquantitatively scored into 0 : negative, 1: 2-4/HPF, 2: 5-9/HPF, 3: 10-20/HPF and 4: $>20 / \mathrm{HPF}$. Acanthocytes were scored for presence/absence.

\subsection{Renal Histopathology}

Two renal pathologists (SH and PS) independently evaluated the kidney biopsies and were blinded to data analysis. Within a renal biopsy specimen, each glomerulus was scored separately for the presence of necrosis, crescents and global glomerular sclerosis. Consequently, the percentage of glomeruli with any of these features was calculated as a fraction of the total number of glomeruli in each renal biopsy. Based on these scorings, 
histopathological subgroupings according to Berden et al. (focal, crescentic, mixed or sclerotic class), and ARRS according to Brix et al. (low, medium or high risk), were performed $[7,28]$. Systematic histological scoring of tubular injury lesions was evaluated as previously described [33]. In brief, epithelial simplification and tubular dilatation, nonisometric cell vacuolization, cellular, red blood cell (RBC), and hyaline casts were given a score between 0 and 4 as a percentage of the total affected cortical area of the biopsy (score $0:<1 \%, 1: \geq 1-10 \%, 2: \geq 10-25 \%, 3: \geq 25-50 \%, 4:>50 \%$ ). Kidney biopsies were also evaluated analogous to the Banff scoring system for allograft pathology [42]. Banff score lesions included interstitial inflammation $(i)$, tubulitis $(t)$, arteritis $(v)$, glomerulitis $(g)$, interstitial fibrosis $(c i)$, tubular atrophy $(c t)$, arteriolar hyalinosis $(a h)$, peritubular capillaritis $(p t c)$, total inflammation $(t i)$, inflammation in areas of IFTA (i-IFTA), and tubulitis in areas of IFTA ( $t$-IFTA). The Banff scoring system had three grades: none (0), mild (1), moderate (2), and severe (3). The cut-off points for $i$ were $<10 \%, 10-25 \%, 26-50 \%$ and $>50 \%$, respectively. Cut-off points for $t$ were $0,1-4,5-10$ and $>10$ mononuclear cells/tubular cross section. Cut-off points for $v$ were no arteritis, mild- to-moderate intimal arteritis in at least 1 arterial cross section, severe intimal arteritis with at least $25 \%$ luminal area lost in at least 1 arterial cross section, transmural arteritis and/or arterial fibrinoid change, and medial smooth muscle necrosis with lymphocytic infiltrate in vessel, respectively. Cut-off points for $g$ were no glomerulitis, segmental or global glomerulitis in less than $25 \%$ of glomeruli, segmental or global glomerulitis in 25 to $75 \%$ of glomeruli, and segmental or global glomerulitis in more than $75 \%$ of glomeruli. Cut-off points for $c i$ were interstitial fibrosis in up to $5 \%, 6-25 \%, 26-50 \%$ and $>50 \%$ of cortical area. Cut-off points for $c t$ were no tubular atrophy, and tubular atrophy involving up to $25 \%, 26-50 \%$ and $>50 \%$ of the area of cortical tubules. Cut-off points for ah were no PAS-positive hyaline arteriolar thickening, mild-to-moderate PAS-positive hyaline thickening in at least 1 arteriole, in more than 1 arteriole, and in many arterioles. Cut-off points for $p t c$ were a maximum number of leukocytes $<3$, at least 1 leukocyte cell in $\geq 10 \%$ of cortical peritubular capillaries (PTCs) with 3-4 leukocytes in most severely involved PTC, at least 1 leukocyte in $\geq 10 \%$ of cortical PTC with 5-10 leukocytes in most severely involved PTC, and at least 1 leukocyte in $\geq 10 \%$ of cortical PTC with $>10$ leukocytes in most severely involved PTC. Cut-off points for $t i$ were $<10 \%, 10-25 \%, 26-50 \%$ and $>50 \%$ of total cortical parenchyma inflamed. Cut-off points for $i$-IFTA and $t$-IFTA were no inflammation or less than $10 \%, 10-25 \%, 26-50 \%$ and $>50 \%$ of scarred cortical parenchyma.

\subsection{Statistical Methods}

Variables were tested for normal distribution using the Shapiro-Wilk test. Nonnormally distributed continuous variables are expressed as median and interquartile range (IQR), and categorical variables are presented as frequency and percentage. Statistical comparisons were not formally powered or prespecified. For group comparisons, the MannWhitney U-test was used to determine differences in medians. Nonparametric betweengroup comparisons were performed with Pearson's Chi-square test. Spearman's correlation was performed to assess the correlation between clinical, laboratory, and histopathological parameters, and heatmaps reflecting the mean values of Spearman's $\rho$ are shown, the asterisks indicating significant correlations. Data analyses were performed with GraphPad Prism (version 9.1.1 for MacOS, GraphPad Software, San Diego, CA, USA).

\section{Results}

\subsection{Description of Study Population}

A total number of 53 renal biopsies with confirmed renal involvement of AAV were identified. Among them, complete systematic histological scoring of tubular injury lesions was available for $48 / 53(90.6 \%)$ renal biopsies (Figure 1). Histopathological subgroupings revealed 16/48 (33.3\%) crescentic, $23 / 48$ (47.9) focal, $3 / 48(6.3 \%)$ sclerotic, and 6/48 (12.5\%) mixed class ANCA GN [7]. ARRS was high in $8 / 48(16.7 \%)$, medium in 22/48 (45.8\%), and low risk class ANCA GN in 18/48 (37.5\%) cases (Figure 1) [28]. 


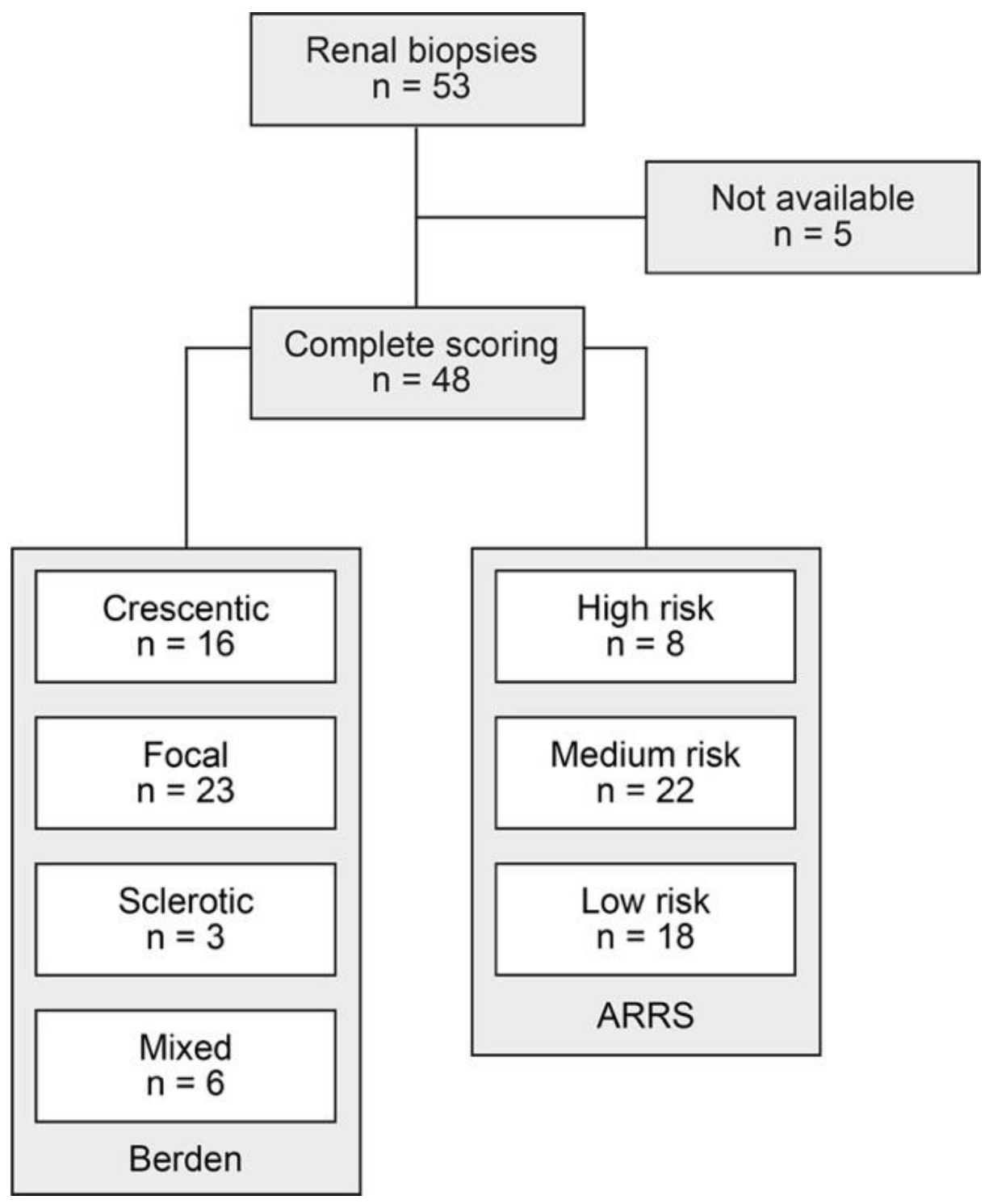

Figure 1. Total patient cohort of ANCA GN: STROBE flow chart of patient disposition with systematic histological scoring of tubular injury lesions. Abbreviations: ANCA, anti-neutrophil cytoplasmic antibodies; ARRS, ANCA renal risk score; GN, glomerulonephritis; STROBE, Strengthening the Reporting of Observational Studies in Epidemiology.

The baseline characteristics of the entire cohort are shown in Table 1. In this cohort, the median (IQR) age at diagnosis was 63 (54.25-74) years, 19/48 (39.6) were female, and all were Caucasian. Disease onset was 18 (7-46) days before admission, and kidney biopsy was performed 6 (3-9.5) days after admission to confirm the renal involvement of AAV. There were $26 / 48(54.2 \%)$ positive for myeloperoxidase (MPO) and 22/48 (45.8\%) positive for proteinase 3 (PR3) ANCA. There were 26/48 (54.2\%) patients categorized as MPA and the remainder as GPA. The majority of patients $(85.4 \%)$ had a new diagnosis of AAV. There were 39/48 (81.3) patients with extrarenal manifestation of AAV (28 with lung, 8 with sinus, 10 with joint, 4 with ear, 3 with eye, 5 with peripheral nerve, and 8 with skin involvement), and $5 / 48(10.4 \%)$ had alveolar hemorrhage. The worst median (IQR) eGFR at disease onset was $17.25(9.525-48.15) \mathrm{mL} / \mathrm{min} / 1.73 \mathrm{~m}^{2}$, and $16 / 48$ (33.3) required dialysis within 30 days after admission. Tubular injury lesions were detectable in a total number of $46 / 48(95.8 \%)$ ANCA GN cases (Table 1). 
Table 1. Clinical and laboratory parameters of the total ANCA GN cohort.

\begin{tabular}{|c|c|}
\hline Parameter & Value \\
\hline Median age (IQR)-years & $63(54.25-74)$ \\
\hline Female sex-no. $(\%)$ & $19(39.58)$ \\
\hline Disease onset-days before admission (IQR) & $18(7-46)$ \\
\hline Kidney biopsy - days after admission (IQR) & $6(3-9.5)$ \\
\hline ANCA subtype MPA/GPA-no. (\%) & $26(54.2) / 22(45.8)$ \\
\hline History of vasculitis-no. (\%) & $7(14.6)$ \\
\hline Dialysis within 30 days after admission-no. (\%) & $16(33.3)$ \\
\hline Median BVAS (IQR)-points & $18(15-20.75)$ \\
\hline Extrarenal manifestation-no. $(\%)$ & $39(81.3)$ \\
\hline Lung involvement-no. (\%) & $28(58.3)$ \\
\hline Pulmonary hemorrhage-no. (\%) & $5(10.4)$ \\
\hline Sinus involvement-no. (\%) & $8(16.7)$ \\
\hline Joint involvement-no. (\%) & $10(20.8)$ \\
\hline Ear involvement-no. $(\%)$ & $4(8.3)$ \\
\hline Eye involvement-no. (\%) & $3(6.3)$ \\
\hline Nerve involvement-no. (\%) & $5(10.4)$ \\
\hline Skin involvement-no. (\%) & $8(16.7)$ \\
\hline ANCA subtype MPO/PR3-no. (\%) & $26(54.2)$ \\
\hline Serum creatinine (IQR)—mg/dL & $3.155(1.355-5.138)$ \\
\hline $\mathrm{eGFR}(\mathrm{IQR})-\mathrm{mL} / \mathrm{min} / 1.73 \mathrm{~m}^{2}$ & $17.25(9.525-48.15)$ \\
\hline $\mathrm{CRP}(\mathrm{IQR})-\mathrm{mg} / \mathrm{L}$ & $63.7(22.38-108)$ \\
\hline $\mathrm{C} 3 \mathrm{c}(\mathrm{IQR})-\mathrm{g} / \mathrm{L}$ & $1.295(1.035-1.408)$ \\
\hline $\mathrm{C} 4(\mathrm{IQR})-\mathrm{g} / \mathrm{L}$ & $0.26(0.2-0.3075)$ \\
\hline $\mathrm{uPCR}(\mathrm{IQR})-\mathrm{mg} / \mathrm{g}$ & 1067 (523.6-2038) \\
\hline $\mathrm{uACR}(\mathrm{IQR})-\mathrm{mg} / \mathrm{g}$ & $451.4(192.4-979.1)$ \\
\hline$\alpha_{1}$-microglobulin (IQR)-mg/g & $69.67(34.85-186)$ \\
\hline$\alpha_{2}$-macroglobulin (IQR)-mg/g & $5.055(2.903-12.42)$ \\
\hline $\operatorname{IgG}(\mathrm{IQR})-\mathrm{mg} / \mathrm{g}$ & $56.52(21.63-209.5)$ \\
\hline Leukocyturia (IQR)—per HPF & $3(2-4)$ \\
\hline Hematuria (IQR)—per HPF & $4(3.25-4)$ \\
\hline Acanthocytes-no. (\%) & $7(14.6)$ \\
\hline Crescentic class-no. (\%) & $16(33.3)$ \\
\hline Focal class-no. (\%) & $23(47.9)$ \\
\hline Sclerotic class-no. $(\%)$ & $3(6.3)$ \\
\hline Mixed class-no. $(\%)$ & $6(12.5)$ \\
\hline ARRS high risk-no. (\%) & $8(16.7)$ \\
\hline ARRS medium risk-no. (\%) & $22(45.8)$ \\
\hline ARRS low risk-no. (\%) & $18(16.7)$ \\
\hline Any lesion-no. (\%) & $46 / 48(95.8)$ \\
\hline Tubular dilatation-no. (\%) & $44 / 48(91.7)$ \\
\hline Vacuolization-no. (\%) & $9 / 48(18.8)$ \\
\hline Cellular casts-no. $(\%)$ & $19 / 48(39.6)$ \\
\hline RBC casts-no. (\%) & $21 / 48(43.8)$ \\
\hline Hyaline casts-no. $(\%)$ & $28 / 48(58.3)$ \\
\hline
\end{tabular}

Continuous variables are expressed as median and interquartile range (IQR), categorical variables are presented as frequency and percentage. Abbreviations: ANCA, anti-neutrophil cytoplasmic antibodies; BVAS, Birmingham Vasculitis Activity Score; C3c, complement factor 3 conversion product; C4, complement factor 4; CRP, C-reactive protein; eGFR, estimated glomerular filtration rate (CKD-EPI); GN, glomerulonephritis; GPA, granulomatosis with polyangiitis; IQR, interquartile range; MPA, microscopic polyangiitis; MPO, myeloperoxidase; no., number; PR3, proteinase 3; RBC, red blood cell; uPCR, urinary protein-to-creatinine ratio; uACR, urinary albumin-tocreatinine ratio.

Systematic histological scoring of tubular changes revealed that tubular epithelial simplification and dilatation was present in $44 / 48(91.7 \%)$ of ANCA GN, epithelial vacuolization in $9 / 48(18.8 \%)$, cellular casts in 19/48 (60.4\%), red blood cell (RBC) casts in $21 / 48(43.8 \%)$, and hyaline casts in 28/48 (58.3\%) of cases (Figure 2 and Table 1), with 

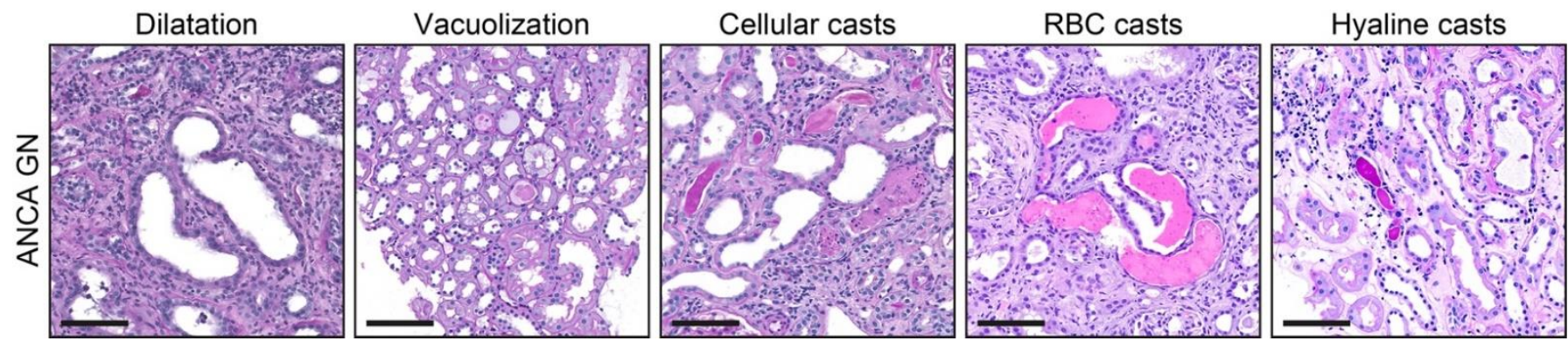

Figure 2. Tubular injury lesions in ANCA GN: Representative photomicrographs with tubular injury lesions are shown (scale bars: $100 \mu \mathrm{m}$ ). Abbreviations: ANCA, anti-neutrophil cytoplasmic antibodies; GN, glomerulonephritis; RBC, red blood cell.

Table 2. Systematic scoring of tubular injury lesions in the total ANCA GN cohort.

\begin{tabular}{cccccc}
\hline Tubular Injury Lesion & $\mathbf{0}$ & $\mathbf{1}$ & $\mathbf{2}$ & $\mathbf{3}$ & $\mathbf{4}$ \\
\hline Tubular dilatation-no. (\%) & $4(8.3)$ & $7(14.6)$ & $11(22.9)$ & $10(20.8)$ & $16(33.3)$ \\
Vacuolization-no. (\%) & $39(81.3)$ & $9(18.8)$ & $0(0)$ & $0(0)$ & $0(0)$ \\
Cellular casts-no. (\%) & $19(39.6)$ & $24(50)$ & $4(8.3)$ & $0(0)$ & $1(2.1)$ \\
RBC casts-no. (\%) & $27(56.3)$ & $17(35.4)$ & $4(8.3)$ & $0(0)$ & $0(0)$ \\
Hyaline casts-no. (\%) & $20(41.7)$ & $21(43.8)$ & $6(12.5)$ & $1(2.1)$ & $0(0)$ \\
\hline
\end{tabular}

Abbreviations: ANCA, anti-neutrophil cytoplasmic antibodies; GN, glomerulonephritis; no., number; RBC, red blood cell.

To elucidate the association between tubular injury lesions in ANCA GN, we next analyzed the association of distinct lesions among each other. Interestingly, we did not find any association between different tubular injury lesions (Figure 3), implicating that presence of each lesion reflected a distinct tubular injury.

\subsection{Distinct Clinical Parameters and Laboratory Markers Associate with Tubular Injury Lesions in $A A V$}

We first analyzed tubular injury lesions in association with clinical parameters, laboratory systemic, and urinary markers in AAV. Tubular epithelial simplification and dilatation correlated with the MPA subtype, epithelial vacuolization with systemic disease activity reflected by BVAS not attributed to a specific extrarenal manifestation, and hyaline casts with a history of AAV and less sinus involvement (Figure 4A). Among systematic laboratory parameters, tubular dilatation associated with MPO subtype, C3c hypocomplementemia, severe renal involvement, and urinary albumin-to-creatinine ratio (uACR, Figure 4B). Cellular casts correlated with hematuria, detection of urinary acanthocytes, and tubular proteinuria reflected by urinary $\alpha_{1}$-microglobulin (Figure $4 B$ ). Finally, RBC casts were associated with increased levels of $C$-reactive protein (CRP), leukocyturia, and hematuria (Figure 4B), implicating that RBC casts represent active glomerular disease and subsequent glomerular hemorrhage. In summary, systematic scoring of tubular injury patterns revealed distinct clinical parameters and laboratory markers in association with tubular lesions in AAV.

\subsection{Acute Tubular Injury Patterns Correlate with Active ANCA GN and Tubular Lesions Reflecting Later Stages of Kidney Disease Correlated with Chronic Glomerular Lesions}

Next, we directly correlated the tubular injury lesions with the histopathological findings in ANCA GN, including glomerular and tubulointerstitial injury. Tubular dilatation was associated with a reduced number of normal glomeruli and ARRS low risk categoriza- 
tion (Figure 5A). RBC casts correlated with active glomerular disease, reflected by necrosis, crescents, and a reduced fraction of glomeruli affected by global glomerular sclerosis (Figure 5A), further corroborating that RBC casts represent active glomerular disease. This was also reflected by categorization into the less mixed class, and was predominant in the crescentic class ANCA GN (Figure 5A). In contrast, hyaline casts were associated with an increased fraction of glomeruli with global glomerular sclerosis (Figure 5A). Interestingly, no interstitial lesion, despite renal fibrosis, correlated with any pattern of tubular injury, implicating that distinct tubular lesions reflected nephron damage, rather than interstitial injury (Figure 5B). In summary, acute tubular injury patterns correlated with active ANCA $\mathrm{GN}$, whereas tubular lesions reflecting later stages of kidney disease correlated with chronic glomerular lesions.

\section{Spearman's $\rho$}

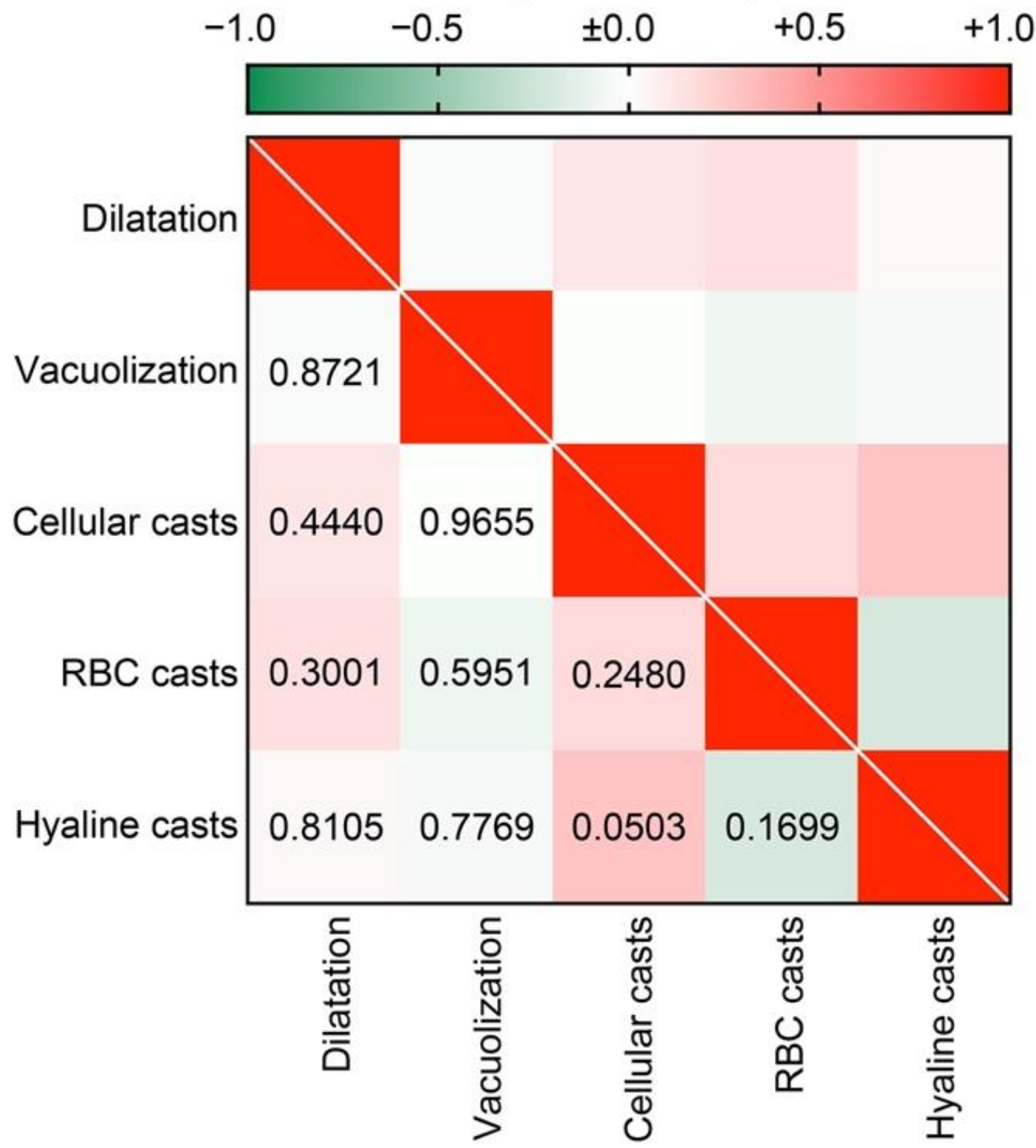

Figure 3. Direct association between tubular injury lesions in AAV: Association between distinct tubular injury lesions are shown by heatmap reflecting mean values of Spearman's $\rho$, values of $p$ are shown for each association. Abbreviations: AAV, ANCA-associated vasculitis; RBC, red blood cell. 
A

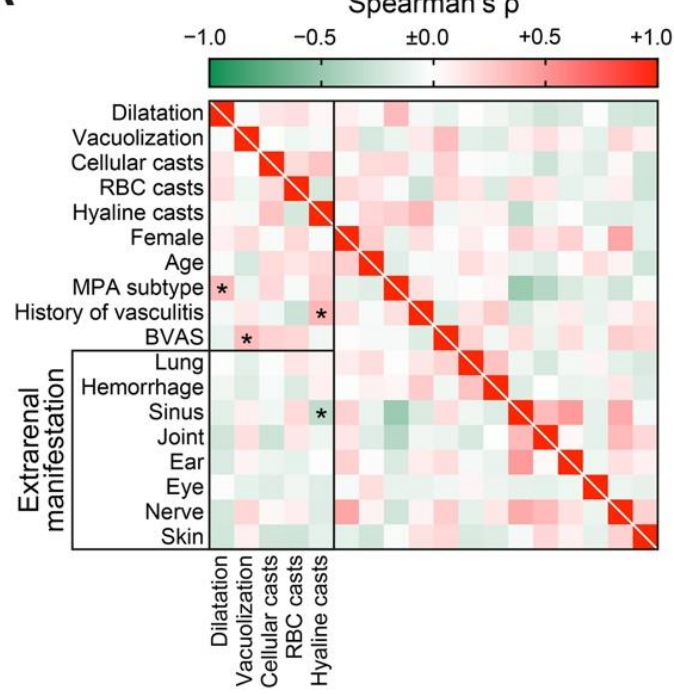

B

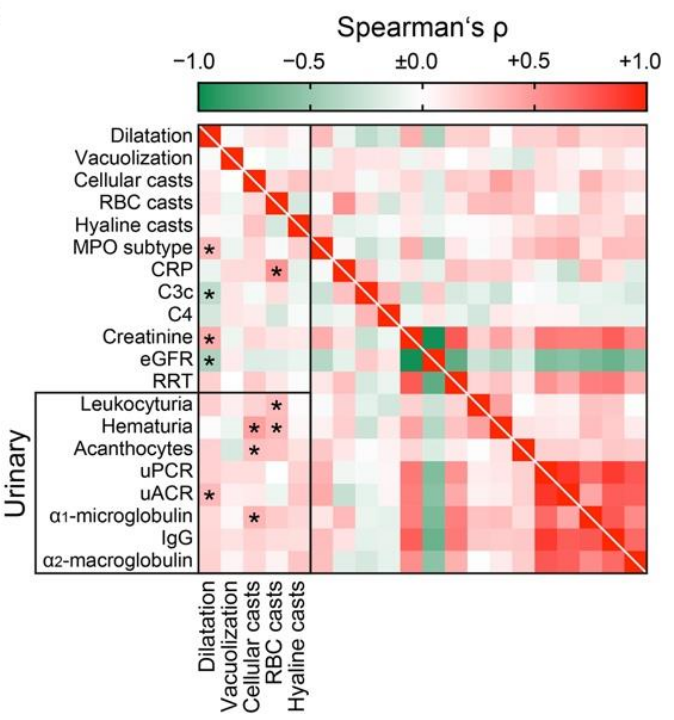

Figure 4. Distinct clinical parameters and laboratory markers associate with tubular injury lesions in AAV: (A) Association between tubular injury lesions and clinical parameters are shown by heatmap reflecting mean values of Spearman's $\rho$, asterisks indicate $p<0.05$. (B) Association between tubular injury lesions and laboratory markers are shown by heatmap reflecting mean values of Spearman's $\rho$, asterisks indicate $p<0.05$. Abbreviations: AAV, ANCA-associated vasculitis; BVAS, Birmingham Vasculitis Activity Score; C3c, complement factor 3 conversion product; C4, complement factor 4; CRP, C-reactive protein; eGFR, glomerular filtration rate (CKD-EPI); GN, glomerulonephritis; IgG, immunoglobulin G; MPA, microscopic polyangiitis; MPO, myeloperoxidase; RBC, red blood cell; RRT, renal replacement therapy; uACR, urinary albumin-to-creatinine ratio, uPCR, urinary protein-to-creatinine ratio.

A

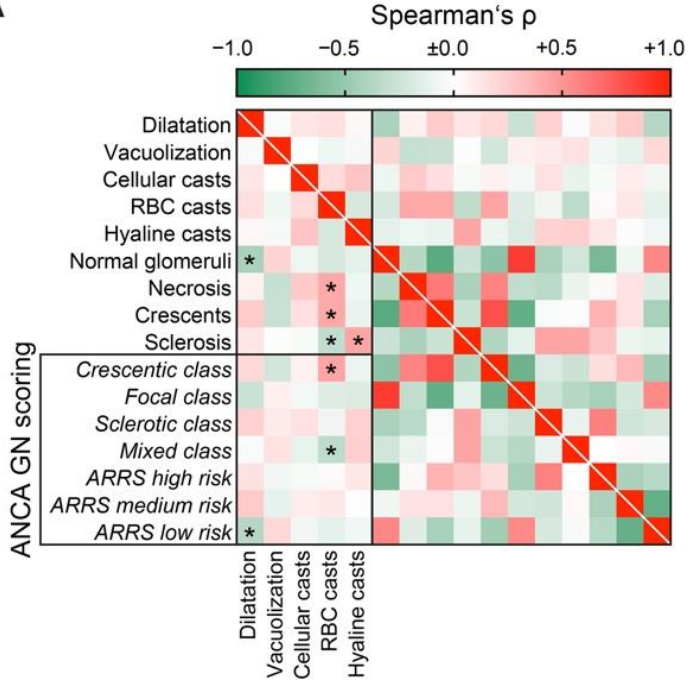

B

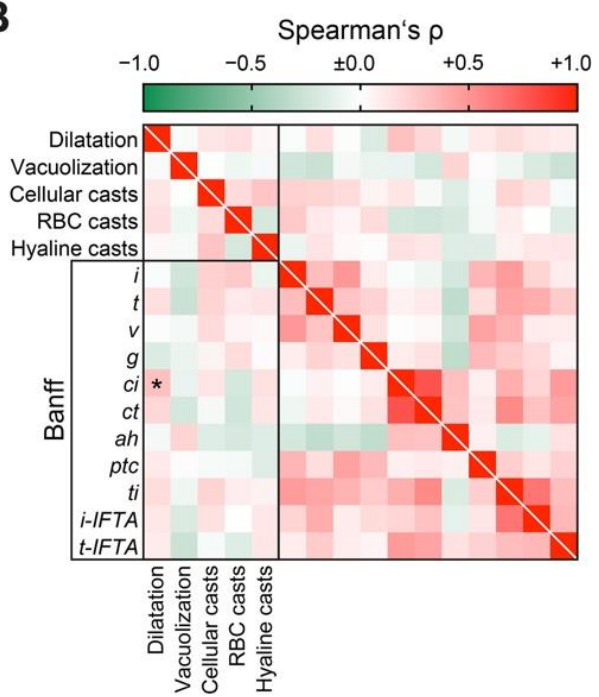

Figure 5. Acute tubular injury patterns correlate with active ANCA GN, whereas tubular lesions reflecting later stages of kidney disease correlated with chronic glomerular lesions: (A) Association between tubular injury lesions and histopathological, findings are shown by heatmap reflecting mean values of Spearman's $\rho$, asterisks indicate $p<0.05$. (B) Association between tubular injury and interstitial lesions analogous to the Banff scoring system are shown by heatmap reflecting mean values of Spearman's $\rho$, asterisks indicate $p<0.05$. Abbreviations: ah, arteriolar hyalinosis; ANCA, anti-neutrophil cytoplasmic antibodies; $c i$, interstitial fibrosis; $c t$, tubular atrophy; $g$, glomerulitis; GN, glomerulonephritis; $i$, interstitial inflammation; $i$-IFTA, inflammation in IFTA; $t$, tubulitis; $p t c$, peritubular capillaritis; RBC, red blood cell; $t i$, total inflammation; $t$-IFTA, tubulitis in IFTA; $v$, intimal arteritis. 


\section{Discussion}

Acute tubular injury was characterized by distinct histological changes, with the loss of brush borders, cytoplasmic vacuolization, and fragmentation with detachment of tubular cells into the lumen producing obstructive casts [43,44]. Finally, severe tubular injury also contributed to epithelial cell apoptosis and necrosis [45]. As a result of persistent tubular injury, TA/IF develops and further impairs kidney function, characterized by a vicious circle of tubular epithelial damage, TA/IF, and chronic glomerular injury [31,32]. Elucidating the molecular alterations that occur in association with distinct tubular injury lesions are of great importance to understand the mechanisms that contribute to acute and chronic kidney damage. Therefore, the determination of the severity, type, and temporal context of injury can also determine molecular responses and kidney damage. Types of injury determines damage to distinct compartments in the kidney, including different nephrons or even segments [46]. In addition, a type of injury can mediate the same or different transcriptional alterations depending on the injured compartment or cellular entity [47]. Therefore, a systematic analysis of injuries to different renal compartments, and their association among each other, is crucial to identify the interplay in response to kidney injury.

We have expanded our current knowledge of distinct tubular injury lesions by systematic scoring of tubular, glomerular, and interstitial lesions in ANCA GN. We have confirmed that tubular injury lesions in renal biopsies are frequently observed in the majority of cases with ANCA GN. Furthermore, a systematic histological scoring of tubular injury patterns revealed distinct clinical parameters and laboratory markers in association with tubular lesions in AAV. Finally, acute tubular injury patterns correlated with active ANCA GN, reflected by glomerular necrosis and crescents, whereas tubular lesions reflecting later stages of kidney disease correlated with chronic glomerular lesions, reflected by global glomerular sclerosis. We have shown that tubular epithelial simplification with dilatation correlated with MPA and MPO subtypes, C3c hypocomplementemia, severe renal involvement, and $\mathrm{uACR}$. This observation is of interest since MPA and MPO subtypes have previously been associated with more severe acute and chronic renal damage $[35,48]$. Tubular dilatation is observed after severe kidney injury and has initially been attributed to tubular obstruction [49-51]. However, more recent studies revealed that tubular dilatation occurs in the recovery phase of kidney injury, independent of tubular obstruction [52]. Hyperplasia of regenerating, dividing tubular epithelial cells and abnormalities of the tubular basement membrane, contributes to tubular dilatation during kidney repair, finally resolving after kidney repair [52]. Our observations that tubular dilatation correlated with severe kidney injury was in line with this concept. However, we also observed that tubular dilatation was associated with a reduced fraction of normal glomeruli and interstitial fibrosis. In this context, dilated tubules, along with interstitial fibrosis, have previously been considered as evidence of advanced stages of kidney disease [53,54]. Furthermore, we have shown that $\mathrm{RBC}$ casts were associated with increased levels of C-reactive protein (CRP), leukocyturia, and hematuria, confirming the concept that RBC casts represent active glomerular disease and subsequent glomerular hemorrhage [55]. This is further supported by the correlation between RBC casts and active glomerular disease reflected by necrosis, crescents, and a reduced fraction of glomeruli affected by global glomerular sclerosis. RBC casts are long known, and have been described in various kidney diseases [56]. In patients with systemic vasculitis, $\mathrm{RBC}$ casts due to glomerular hemorrhage are considered a marker of active renal disease [55]. Erythrocytes that pass through the glomerular basement membrane gaps are merged with uromodulin produced in the Henle's loop and from RBC casts that are excreted into urine [57]. Finally, we found that hyaline casts were associated with an increased fraction of glomeruli with global glomerular sclerosis. Hyaline casts reflect nephron obstruction in the final stage of tubular degeneration, due to renal stasis or nephron obstruction [57]. This concept was further supported by our observation that no interstitial lesion, despite renal fibrosis, correlated with any acute tubular injury pattern, implicating that distinct tubular lesions reflect nephron damage, rather than interstitial injury. 
The main limitations of our study were its retrospective design, the small patient number, and lack of data on long-term renal survival. Nevertheless, we have provided a systematic, histological analysis of tubular injury lesions in ANCA GN frequently observed in the majority of cases with AAV, and have provided evidence for an interplay between different renal compartments.

\section{Conclusions}

To our knowledge, this is the first report of systematic scoring of acute tubular injury patterns in ANCA GN. Acute tubular injury patterns correlated with active ANCA GN, whereas tubular lesions reflecting later stages of kidney disease correlated with chronic glomerular lesions.

Author Contributions: B.T. conceived the study, collected and analyzed data and wrote the first draft. S.H. and D.T. collected and analyzed data. S.H. and P.S. evaluated histopathological findings. P.K. analyzed data and edited the manuscript. All authors have read and agreed to the published version of the manuscript.

Funding: This research was funded by the Research program, University Medical Center, University of Göttingen, grant number 1403720. This research was also funded by the German Research Foundation, KFO (CRU) 5002, grant number STR 638/3-1 (DFG). We also acknowledge support by the Open Access Publication Funds of the Göttingen University.

Institutional Review Board Statement: The study was conducted according to the guidelines of the Declaration of Helsinki, and approved by the Institutional Review Board of the University Medical Center Göttingen, Germany (protocol code: 22/2/14, approval date 22 September 2014 and 28/09/17, approval date 17 November 2017). The patients/participants provided their written informed consent to participate in this study.

Informed Consent Statement: Informed consent was obtained from all subjects involved in the study.

Data Availability Statement: Deidentified data are available on reasonable request from the corresponding author.

Acknowledgments: The authors thank Ulrike Ehbrecht for technical assistance.

Conflicts of Interest: The authors declare no conflict of interest. The funders had no role in the design of the study; in the collection, analyses, or interpretation of data; in the writing of the manuscript, or in the decision to publish the results.

\section{References}

1. Jennette, J.C.; Falk, R.J.; Bacon, P.A.; Basu, N.; Cid, M.C.; Ferrario, F.; Flores-Suarez, L.F.; Gross, W.L.; Guillevin, L.; Hagen, E.C.; et al. 2012 Revised International Chapel Hill Consensus Conference Nomenclature of Vasculitides. Arthritis Rheum. 2013, 65, 1-11. [CrossRef]

2. Hruskova, Z.; Stel, V.S.; Jayne, D.; Aasarød, K.; De Meester, J.; Ekstrand, A.; Eller, K.; Heaf, J.G.; Hoitsma, A.; Jimenéz, C.M.; et al. Characteristics and Outcomes of Granulomatosis with Polyangiitis (Wegener) and Microscopic Polyangiitis Requiring Renal Replacement Therapy: Results From the European Renal Association-European Dialysis and Transplant Association Registry. Am. J. Kidney Dis. 2015, 66, 613-620. [CrossRef]

3. Bajema, I.M.; Hagen, E.C.; Hermans, J.; Noël, L.-H.; Waldherr, R.; Ferrario, F.; van der Woude, F.J.; Bruijn, J.A. Kidney biopsy as a predictor for renal outcome in ANCA-associated necrotizing glomerulonephritis. Kidney Int. 1999, 56, 1751-1758. [CrossRef]

4. Hauer, H.A.; Bajema, I.M.; Van Houwelingen, H.C.; Ferrario, F.; Noël, L.-H.; Waldherr, R.; Jayne, D.R.; Rasmussen, N.; Bruijn, J.A.; Hagen, E.C. Determinants of outcome in ANCA-associated glomerulonephritis: A prospective clinico-histopathological analysis of 96 patients. Kidney Int. 2002, 62, 1732-1742. [CrossRef] [PubMed]

5. de Lind van Wijngaarden, R.A.; Hauer, H.A.; Wolterbeek, R.; Jayne, D.R.; Gaskin, G.; Rasmussen, N.; Noël, L.-H.; Ferrario, F.; Waldherr, R.; Hagen, E.C.; et al. Clinical and Histologic Determinants of Renal Outcome in ANCA-Associated Vasculitis: A Prospective Analysis of 100 Patients with Severe Renal Involvement. J. Am. Soc. Nephrol. 2006, 17, 2264-2274. [CrossRef] [PubMed]

6. Bajema, I.M.; Hagen, E.C.; Hansen, B.E.; Waldherr, R.; Van Der Woude, F.J.; Bruijn, J.A.; Hermans, J.; Noël, L.H.; Ferrario, F. The renal histopathology in systemic vasculitis: An international survey study of inter- and intra-observer agreement. Nephrol. Dial. Transplant. 1996, 11, 1989-1995. [CrossRef] [PubMed] 
7. Berden, A.E.; Ferrario, F.; Hagen, E.C.; Jayne, D.R.; Jennette, J.C.; Joh, K.; Neumann, I.; Noël, L.-H.; Pusey, C.D.; Waldherr, R.; et al. Histopathologic Classification of ANCA-Associated Glomerulonephritis. J. Am. Soc. Nephrol. 2010, 21, 1628-1636. [CrossRef] [PubMed]

8. Chang, D.-Y.; Wu, L.-H.; Liu, G.; Chen, M.; Kallenberg, C.G.M.; Zhao, M.-H. Re-evaluation of the histopathologic classification of ANCA-associated glomerulonephritis: A study of 121 patients in a single center. Nephrol. Dial. Transplant. 2012, 27, 2343-2349. [CrossRef]

9. Ellis, C.L.; Manno, R.L.; Havill, J.P.; Racusen, L.C.; Geetha, D. Validation of the new classification of pauci-immune glomerulonephritis in a United States cohort and its correlation with renal outcome. BMC Nephrol. 2013, 14, 210. [CrossRef]

10. Togashi, M.; Komatsuda, A.; Nara, M.; Omokawa, A.; Okuyama, S.; Sawada, K.; Wakui, H. Validation of the 2010 histopathological classification of ANCA-associated glomerulonephritis in a Japanese single-center cohort. Mod. Rheumatol. 2014, $24,300-303$. [CrossRef]

11. Rahmattulla, C.; Bruijn, J.A.; Bajema, I.M. Histopathological classification of antineutrophil cytoplasmic antibody-associated glomerulonephritis: An update. Curr. Opin. Nephrol. Hypertens. 2014, 23, 224-231. [CrossRef]

12. Lee, T.; Gasim, A.; Derebail, V.K.; Chung, Y.; McGregor, J.G.; Lionaki, S.; Poulton, C.J.; Hogan, S.L.; Jennette, J.C.; Falk, R.J.; et al. Predictors of Treatment Outcomes in ANCA-Associated Vasculitis with Severe Kidney Failure. Clin. J. Am. Soc. Nephrol. 2014, 9, 905-913. [CrossRef]

13. Diaz-Crespo, F.; Villacorta, J.; Acevedo, M.; Cavero, T.; Guerrero, C.; Díaz, E.G.; Orradre, J.L.; Martinez, M.A.; Praga, M.; Fernandez-Juarez, G. The predictive value of kidney biopsy in renal vasculitis: A multicenter cohort study. Hum. Pathol. 2016, 52, 119-127. [CrossRef]

14. Nohr, E.; Girard, L.; James, M.; Benediktsson, H. Validation of a histopathologic classification scheme for antineutrophil cytoplasmic antibody-associated glomerulonephritis. Hum. Pathol. 2014, 45, 1423-1429. [CrossRef]

15. Noone, D.G.; Twilt, M.; Hayes, W.N.; Thorner, P.S.; Benseler, S.; Laxer, R.M.; Parekh, R.S.; Hebert, D. The New Histopathologic Classification of ANCA-Associated GN and Its Association with Renal Outcomes in Childhood. Clin. J. Am. Soc. Nephrol. 2014, 9 , 1684-1691. [CrossRef]

16. Quintana, L.F.; Peréz, N.S.; De Sousa, E.; Rodas, L.M.; Griffiths, M.H.; Solé, M.; Jayne, D. ANCA serotype and histopathological classification for the prediction of renal outcome in ANCA-associated glomerulonephritis. Nephrol. Dial. Transplant. 2014, 29, 1764-1769. [CrossRef] [PubMed]

17. Hilhorst, M.; Wilde, B.; Vriesman, P.V.B.; Van Paassen, P.; Tervaert, J.W.C. Estimating Renal Survival Using the ANCA-Associated GN Classification. J. Am. Soc. Nephrol. 2013, 24, 1371-1375. [CrossRef]

18. Iwakiri, T.; Fujimoto, S.; Kitagawa, K.; Furuichi, K.; Yamahana, J.; Matsuura, Y.; Yamashita, A.; Uezono, S.; Shimao, Y.; Hisanaga, S.; et al. Validation of a newly proposed histopathological classification in Japanese patients with anti-neutrophil cytoplasmic antibody-associated glomerulonephritis. BMC Nephrol. 2013, 14, 125. [CrossRef] [PubMed]

19. Ford, S.L.; Polkinghorne, K.; Longano, A.; Dowling, J.; Dayan, S.; Kerr, P.G.; Holdsworth, S.R.; Kitching, A.R.; Summers, S.A. Histopathologic and Clinical Predictors of Kidney Outcomes in ANCA-Associated Vasculitis. Am. J. Kidney Dis. 2014, 63, 227-235. [CrossRef]

20. Naidu, G.; Sharma, A.; Nada, R.; Kohli, H.; Jha, V.; Gupta, K.L.; Sakhuja, V.; Rathi, M. Histopathological classification of pauci-immune glomerulonephritis and its impact on outcome. Rheumatol. Int. 2014, 34, 1721-1727. [CrossRef] [PubMed]

21. Moroni, G.; Binda, V.; Leoni, A.; Raffiotta, F.; Quaglini, S.; Banfi, G.; Messa, P. Predictors of renal survival in ANCA-associated vasculitis. Validation of a histopatological classification schema and review of the literature. Clin. Exp. Rheumatol. 2015, 33, 56-63.

22. Andreiana, I.; Stancu, S.; Avram, A.; Taran, L.; Mircescu, G. ANCA positive crescentic glomerulonephritis outcome in a Central East European cohort: A retrospective study. BMC Nephrol. 2015, 16, 90. [CrossRef] [PubMed]

23. Tanna, A.; Guarino, L.; Tam, F.W.; Rodriquez-Cubillo, B.; Levy, J.B.; Cairns, T.D.; Griffith, M.; Tarzi, R.M.; Caplin, B.; Salama, A.D.; et al. Long-term outcome of anti-neutrophil cytoplasm antibody-associated glomerulonephritis: Evaluation of the international histological classification and other prognostic factors. Nephrol. Dial. Transplant. 2015, 30, 1185-1192. [CrossRef] [PubMed]

24. Córdova-Sánchez, B.M.; Mejía-Vilet, J.M.; Morales-Buenrostro, L.E.; Loyola-Rodríguez, G.; Uribe-Uribe, N.O.; Correa-Rotter, R. Clinical presentation and outcome prediction of clinical, serological, and histopathological classification schemes in ANCAassociated vasculitis with renal involvement. Clin. Rheumatol. 2016, 35, 1805-1816. [CrossRef]

25. Van Daalen, E.; Ferrario, F.; Noël, L.H.; Waldherr, R.; Hagen, E.C.; Bruijn, J.A.; Bajema, I.M. Twenty-five years of RENHIS: A history of histopathological studies within EUVAS. Nephrol. Dial. Transplant. 2015, 30 (Suppl. S1), i31-i36. [CrossRef]

26. Bjørneklett, R.; Sriskandarajah, S.; Bostad, L. Prognostic Value of Histologic Classification of ANCA-Associated Glomerulonephritis. Clin. J. Am. Soc. Nephrol. 2016, 11, 2159-2167. [CrossRef] [PubMed]

27. Chen, Y.-X.; Xu, J.; Pan, X.-X.; Shen, P.-Y.; Li, X.; Ren, H.; Chen, X.-N.; Ni, L.-Y.; Zhang, W.; Chen, N. Histopathological Classification and Renal Outcome in Patients with Antineutrophil Cytoplasmic Antibodies-associated Renal Vasculitis: A Study of 186 Patients and Metaanalysis. J. Rheumatol. 2016, 44, 304-313. [CrossRef] [PubMed]

28. Brix, S.R.; Noriega, M.; Tennstedt, P.; Vettorazzi, E.; Busch, M.; Nitschke, M.; Jabs, W.J.; Özcan, F.; Wendt, R.; Hausberg, M.; et al. Development and validation of a renal risk score in ANCA-associated glomerulonephritis. Kidney Int. 2018, 94, 1177-1188. [CrossRef] 
29. Vergunst, C.E.; Van Gurp, E.; Hagen, E.; Van Houwelingen, H.C.; Hauer, H.A.; Noël, L.-H.; Waldherr, R.; Ferrario, F.; Van Der Woude, F.J.; Bruijn, J.A.; et al. An index for renal outcome in ANCA-associated glomerulonephritis. Am. J. Kidney Dis. 2003, 41, 532-538. [CrossRef]

30. Wu, L.; Li, X.-Q.; Goyal, T.; Eddy, S.; Kretzler, M.; Ju, W.-J.; Chen, M.; Zhao, M.-H. Urinary epidermal growth factor predicts renal prognosis in antineutrophil cytoplasmic antibody-associated vasculitis. Ann. Rheum. Dis. 2018, 77, 1339-1344. [CrossRef]

31. Adachi, T.; Sugiyama, N.; Yagita, H.; Yokoyama, T. Renal atrophy after ischemia-reperfusion injury depends on massive tubular apoptosis induced by TNFalpha in the later phase. Med. Mol. Morphol. 2014, 47, 213-223. [CrossRef]

32. Ferenbach, D.; Bonventre, J.V. Mechanisms of maladaptive repair after AKI leading to accelerated kidney ageing and CKD. Nat. Rev. Nephrol. 2015, 11, 264-276. [CrossRef]

33. Pieters, T.T.; Falke, L.L.; Nguyen, T.Q.; Verhaar, M.C.; Florquin, S.; Bemelman, F.J.; Kers, J.; Vanhove, T.; Kuypers, D.; Goldschmeding, R.; et al. Histological characteristics of Acute Tubular Injury during Delayed Graft Function predict renal function after renal transplantation. Physiol. Rep. 2019, 7, e14000. [CrossRef]

34. Hakroush, S.; Tampe, D.; Korsten, P.; Ströbel, P.; Zeisberg, M.; Tampe, B. Histopathological Findings Predict Renal Recovery in Severe ANCA-Associated Vasculitis Requiring Intensive Care Treatment. Front. Med. 2021, 7. [CrossRef]

35. Hakroush, S.; Kluge, I.; Ströbel, P.; Korsten, P.; Tampe, D.; Tampe, B. Systematic Histological Scoring Reveals More Prominent Interstitial Inflammation in Myeloperoxidase-ANCA Compared to Proteinase 3-ANCA Glomerulonephritis. J. Clin. Med. 2021, 10, 1231. [CrossRef]

36. Hakroush, S.; Tampe, D.; Korsten, P.; Ströbel, P.; Tampe, B. Bowman's capsule rupture links glomerular damage to tubulointerstitial inflammation in ANCA-associated glomerulonephritis. Clin. Exp. Rheumatol 2021, 39 (Suppl. S129), 27-31.

37. Tampe, D.; Korsten, P.; Ströbel, P.; Hakroush, S.; Tampe, B. Proteinuria Indicates Decreased Normal Glomeruli in ANCAAssociated Glomerulonephritis Independent of Systemic Disease Activity. J. Clin. Med. 2021, 10, 1538. [CrossRef] [PubMed]

38. Levey, A.S.; Stevens, L.A.; Schmid, C.H.; Zhang, Y.L.; Castro, A.F.; Feldman, H.I.; Kusek, J.W.; Eggers, P.; Van Lente, F.; Greene, T.; et al. A new equation to estimate glomerular filtration rate. Ann. Intern. Med. 2009, 150, 604-612. [CrossRef]

39. Mukhtyar, C.; Lee, R.; Brown, D.; Carruthers, D.; Dasgupta, B.; Dubey, S.; Flossmann, O.; Hall, C.; Hollywood, J.; Jayne, D.; et al. Modification and validation of the Birmingham Vasculitis Activity Score (version 3). Ann. Rheum. Dis. 2009, 68, 1827-1832. [CrossRef] [PubMed]

40. Le Gall, J.-R.; Lemeshow, S.; Saulnier, F. A New Simplified Acute Physiology Score (SAPS II) Based on a European/North American Multicenter Study. JAMA 1993, 270, 2957-2963. [CrossRef] [PubMed]

41. Waikar, S.S.; Sabbisetti, V.S.; Bonventre, J.V. Normalization of urinary biomarkers to creatinine during changes in glomerular filtration rate. Kidney Int. 2010, 78, 486-494. [CrossRef]

42. Roufosse, C.; Simmonds, N.; Groningen, M.C.-V.; Haas, M.; Henriksen, K.J.; Horsfield, C.; Loupy, A.; Mengel, M.; PerkowskaPtasińska, A.; Rabant, M.; et al. A 2018 Reference Guide to the Banff Classification of Renal Allograft Pathology. Transplantation 2018, 102, 1795-1814. [CrossRef]

43. Nonclercq, D.; Toubeau, G.; Laurent, G.; Tulkens, P.M.; Heuson-Stiennon, J.A. Tissue injury and repair in the rat kidney after exposure to cisplatin or carboplatin. Exp. Mol. Pathol. 1989, 51, 123-140. [CrossRef]

44. Solez, K.; Racusen, L.C.; Marcussen, N.; Slatnik, I.; Keown, P.; Burdick, J.F.; Olsen, S. Morphology of ischemic acute renal failure, normal function, and cyclosporine toxicity in cyclosporine-treated renal allograft recipients. Kidney Int. 1993, 43, $1058-1067$. [CrossRef]

45. Olsen, S.; Burdick, J.F.; Keown, P.A.; Wallace, A.C.; Racusen, L.C.; Solez, K. Primary acute renal failure ("acute tubular necrosis") in the transplanted kidney: Morphology and pathogenesis. Medicine 1989, 68, 173-187. [CrossRef]

46. Lieberthal, W.; Nigam, S.K. Acute renal failure. I. Relative importance of proximal vs. distal tubular injury. Am. J. Physiol. 1998, 275, 623-631. [CrossRef] [PubMed]

47. Xu, K.; Rosenstiel, P.; Paragas, N.; Hinze, C.; Gao, X.; Shen, T.H.; Werth, M.; Forster, C.; Deng, R.; Bruck, E.; et al. Unique Transcriptional Programs Identify Subtypes of AKI. J. Am. Soc. Nephrol. 2016, 28, 1729-1740. [CrossRef]

48. Sinico, R.A.; Di Toma, L.; Radice, A. Renal involvement in anti-neutrophil cytoplasmic autoantibody associated vasculitis. Autoimmun. Rev. 2013, 12, 477-482. [CrossRef] [PubMed]

49. Finn, W.F.; Chevalier, R.L. Recovery from postischemic acute renal failure in the rat. Kidney Int. 1979, 16, 113-123. [CrossRef]

50. Norlén, B.J.; Engberg, A.; Källskog, Ö.; Wolgast, M. Nephron function of the transplanted rat kidney. Kidney Int. 1978, 14, 10-20. [CrossRef] [PubMed]

51. Oliver, J. Correlations of structure and function and mechanisms of recovery in acute tubular necrosis. Am. J. Med. 1953, 15, 535-557. [CrossRef]

52. Shimizu, A.; Masuda, Y.; Ishizaki, M.; Sugisaki, Y.; Yamanaka, N. Tubular dilatation in the repair process of ischaemic tubular necrosis. Virchows Arch. 1994, 425, 281-290. [CrossRef]

53. Kimura, M.; Suzuki, T.; Hishida, A. A Rat Model of Progressive Chronic Renal Failure Produced by Microembolism. Am. J. Pathol. 1999, 155, 1371-1380. [CrossRef]

54. Remuzzi, G.; Ruggenenti, P.; Benigni, A. Understanding the nature of renal disease progression. Kidney Int. 1997, 51, 2-15. [CrossRef] [PubMed]

55. Winearls, C.G.; Rees, A.J.; Savage, C.O.S.; Evans, D.J.; Lockwood, C.M. Microscopic Polyarteritis: Presentation, Pathology and Prognosis. QJM Int. J. Med. 1985, 56, 467-483. 
56. Beale, L.S. Kidney Diseases, Urinary Deposits, and Calculous Disorders: Their Nature and Treatment; John Churchill and Sons: London, UK, 1869; Volume 43, pp. 370-383.

57. Cavanaugh, C.; Perazella, M.A. Urine Sediment Examination in the Diagnosis and Management of Kidney Disease: Core Curriculum 2019. Am. J. Kidney Dis. 2019, 73, 258-272. [CrossRef] [PubMed] 